\title{
Study on the Development of Colleges in Non-capital Cities
}

\author{
Yue Yuan \\ Institute of Higher Education, Xianyang Normal University, 712000
}

\author{
Keywords: Non-capital cities; Colleges; Status quo; Ldeas
}

\begin{abstract}
In recent years, the rise of local high-level colleges is a new trend in the process of the reform and development of higher education in our country, which has a profound and strong influence on the pattern of higher education in China. Under the guidance of the country's policy of rejuvenating the country through science and education to build a strong higher education of nation, so how to promote the construction of local colleges has become the main theme of the reform and development of higher education in China. The orderly and healthy development of universities in non-capital cities is an important part of the construction of local colleges. This article briefly analyzes the role and development of colleges in non-capital cities. Combined with the actual situation of education and teaching, the paper provided targeted solutions and measures.

According to statistics from related departments, by the year 2018, there were more than 2,200 ordinary colleges and universities in China, of which only 107 were in the charge of the Ministry of Education and other commissions. There were more than 2,100 local ordinary colleges and universities, accounting for more than $95 \%$ of the total. After the reform and opening up, China's higher education undertakings show a rapid development trend. Especially in recent years, with the continuous increase of investment in higher education by the state and local governments, local colleges have entered the fast track of sustainable development under the joint efforts of all sectors of society.

Local colleges and universities can be divided into provincial capital and non-capital city colleges according to their geographical location. Among them, non-capital city colleges are the main body of local colleges. Although non-capital city universities and provincial capital cities have only one word difference, their development level and characteristics are quite different. As a teacher in non-capital city college, the author engaged in education and teaching-related work for many years, and has deep feelings about the problems encountered in the development of universities in non-capital cities. Over the years, due to the working relationship, the author has met with many non-capital city college teachers, and they have conducted in-depth exchanges and discussions on issues such as education and teaching, and development of innovative ideas. Here, the author combines his own work experience and research on relevant theoretical and practical aspects of the results, making a simple analysis and elaboration on the role of non-capital city development in the development of higher education, and the status quo and the development of ideas and countermeasures in non-capital cities, in order to achieve get good suggestions and ideas from other teachers and experts.
\end{abstract}

\section{The Role of Non-capital Cities University Development in the Development of Higher Education}

Non-capital cities have a large number of colleges, and they play an important historical mission in the development of higher education in China. Doing a good job in the reform and universities construction of non-capital cities has important theoretical research on comprehensive, scientific, and systematic guidance for the development of higher education. First, non-capital city colleges undertake most of the basic education functions of higher education in China. Universities in noncapital cities have large volumes, numerous employees and a large number of students. The basic and primary function of higher education is to obey and serve the nation's talent claims. In the new historical period of China's modernization construction, the demand for talents in all walks of life has been significantly improved in both qualitatively and quantitatively. Non-capital city colleges play a major role in this historic task and support the basic functions of higher education. Therefore, 
the development of non-capital city colleges builds the foundation of higher education development. Second, universities in non-capital cities have effectively extended the depth and breadth of China's higher education. Universities in non-capital cities have a wide distribution with a large number of students.

Non-capital city colleges are like nodes, weaving into a large network of higher education and spreading the function of higher education to a deeper and broader level. The smooth realization of the goal of popularization of higher education in our country is closely related to the development of universities in non-capital cities. Finally, the development of universities in non-capital cities provides valuable theoretical and practical experience for the development of higher education in China. The characteristics of universities in non-capital cities have determined that the problems encountered in their development have the dual characteristics of universality and particularity. Universality refers to the common problem of non-capital cities in developing universities in China. Particularity refers to the fact that different non-capital city colleges may cause special problems and diversification due to differences in their local economic and social development levels. Therefore, careful analysis and refinement of the problems encountered in the development of universities in non-capital cities have very important reference value for the development of higher education.

\section{The Development Status of Non-capital Cities Colleges}

It is undeniable that due to the impact of traditional concepts and specific historical reasons, noncapital city colleges have some inherent deficiencies in their development. For example, their own influence is weak, social recognition is relatively low, and there is a lack of competitiveness in the recruitment and promotion of students. The existence of these problems can be effectively resolved in the short term. The problem itself is not terrible. It is terrible that we avoid and ignore the existence of these problems, and even take some unscientific measures to make the problem intensify, and eventually create a vicious circle or even an irreversible situation. At present, the development of colleges in non-capital cities has the following major problems:

Ideas and Concepts. Non-capital city colleges in provincial capital cities have different environments. Non-capital city colleges live at a disadvantage place, and the scope of contact for teachers and students is very limited. They are often unresponsive to the changes and development of society, and obviously lag behind provincial capital city colleges. Over time, they tend to be conservative in their ideology and are accustomed to sticking to conventions so that they cannot change. This is reflected in the schooling of non-capital-capital cities, that is, the idea of scholarship is relatively backward, and the methods and methods are simply obsolete.

Aspects of School Conditions. One of the bottlenecks restricting the development of universities in non-capital cities is fund. In general, non-capital city colleges are limited by their geographical and economic conditions, and local governments have very limited financial support for colleges. This fund is the main source of funding for local colleges. Local colleges as the main force of the expansion of recruiting students, the size of the school and the number of students in the school significantly increased, followed by a shortage of education funding. According to statistics, the average annual growth rate of students in local colleges since 2002 is about $10 \%$, but the corresponding increase in education investment is far below this figure. Compared with ministry colleges, the funding of local colleges is even more inadequate. Although the local colleges are absolutely dominant in terms of quantity, they cannot be compared with the " 211 ", "985", "Firstrate universities" and other institutions in terms of the amount of funds acquired and the importance they attach. In addition to restrictions on scientific research and other conditions, non-capital city colleges have poor financing capabilities. This has resulted in a single source of funding for universities in non-capital cities. Although the school has a long-term construction goal plan in terms of discipline construction and hardware and software input, it often focuses on ensuring that the limited funds are put into the most necessary issues.

Construction of Teacher Team. How to cultivate and build a highly qualified, professional, and stable faculty is a persistent problem that has plagued the healthy development of universities in 
non-capital cities. Universities in non-capital cities have limited funds, and their urban environment is incomparable with provincial capitals. The fact that the level of discipline construction is not high makes the loss of teachers in non-capital city colleges serious. The starting point for many teachers to upgrade their titles is not to further improve teaching. Instead, prepare for the transfer. This kind of loose teacher structure makes it difficult to ensure that education and teaching goals are achieved.

Professionals Settings. Many non-capital cities have blindly tracked hot spots. New majors tend to focus on the so-called current hot spots to attract students and look at other universities. This kind of professional setup obviously lacks overall planning, lacks rigorous argumentation and in-depth market research, which leads to the new professional development appear rather than heat, which is out of touch with the needs of local economic construction, and may eventually develop into a tasteless school discipline. The professional setting should first proceed from the school's planning and actual characteristics, and must not seek to achieve perfection, blindly follow others, or consume limited resources in vain. Some colleges in the professional setting up like a straight-line, no school education features. In a sense, professional settings are set only for the purpose of setting, without any long-term development considerations.

\section{Non-capital City College Development Ideas and Strategies}

In the new period of social history, colleges have played an important role in the process of building a well-to-do society in an all-round way and realizing the Chinese dream. The social value has also been continuously enriched with the improvement of the requirements for personnel training. The reform and development of colleges is an inevitable requirement to fulfill the mission of good responsibilities, to do a good job of personnel training, scientific exploration, social services, and cultural heritage. Non-capital cities colleges should follow the trend and innovate ideas. The author believes that non-capital city colleges in the development should mainly focus on the following aspects:

Scientific Positioning and Clear Development Ideas. First of all, non-capital city colleges must have a clear understanding of their own characteristics of running schools and the advantages of running a school. Secondly, non-capital city colleges need to profoundly reflect on their own problems in terms of personnel training, for example, whether there is a disconnect between the professional setup and the employer's job requirements, which results in the problem that graduates are not capable enough and have low competitiveness in employment; There is a problem of not having a strong sense of service, conflicting with the needs of local economic development; There is a problem of lack of self-containedness, closing doors, and weak awareness of social contribution. Finally, non-capital city colleges must have a clear understanding of development transformation. The transformation that meets the requirements of the times and the goal of personnel training needs to be changed and how it should be changed. To sum up, this issue mainly includes the transition from discipline-based to employment-based; the transformation from cultivating academic talents to applying technical talents; the construction of the faculty from pure academic pursuits to academic and practical dual-regulation transitions; teaching philosophy from knowledge inculcates the transformation of skills-based training; the management system transforms from a planning model to a modern university system.

Non-capital city colleges must have a scientific orientation for their own development and transformation. The transition process should be scientific and standardized, and be full of research and discussion in the early stage, and comprehensively do a good job in the adjustment of the concept of running schools, development ideas, system construction, disciplines, training objectives, teaching models, assessment and evaluation mechanisms, management services, campus culture construction, etc. We plan globally from large aspect and coordinate advancement. In the process of transition, we must also adhere to the principle of problem-oriented. Only by adhering to the problem-oriented approach can we be able to do something, be pragmatic and efficient, and do no virtual work. 
Demonstrate the Characteristics of Running a School. Running out of features is the core way for non-capital city colleges to improve their competitiveness. The characteristic is the development space, but also the development prospects. Only by highlighting the characteristics of running a school can we further consolidate the direction of subject research. In school running, we must follow the guidance of classification, advocate the principle of characteristic development, and improve the overall strength of education and teaching. Strive to compete in the method that we have but other don't, or people have but we are better. The most obvious advantage of running schools with distinctive features is that they can promote the all-round development of discipline construction with breakthroughs in points. Such a school-running idea is not only conducive to the concentration of resources, but also conducive to strengthening the social service functions.

Obeying and Serving Local Economic Development. Non-capital city colleges should further enhance their awareness of local economic development, vigorously promote collaborative innovation, and establish a number of organizations that integrate talent cultivation, discipline construction and social services. Schools should do a good job of investigating and researching, and stick to local economic and development weaknesses as much as possible. For qualified colleges, professional service groups can be formed. For professional service groups, we may most likely contact hotel service professionals. In recent years, many colleges rely on their own educational resources, rely on the characteristics and specialty of the school as a link, and rely on local companies and even foreign companies to form professional service groups. For example, Shanghai took the lead in establishing the Shanghai Business Group with the Shanghai Business School as the leader and the major Shanghai-based business enterprises as the core. There are many such professional service groups, and most have achieved very good social and economic benefits. These professional service groups combine the advantages of their own constituent elements in a clever way to form a community of benefit-sharing.

Transitional Development Requires the Involvement of Government Functions. The transformation and development of universities in non-capital cities requires the strong support and full cooperation of local governments. Only with the full involvement of the local government, the problems encountered by the school in terms of talent team building, school-enterprise cooperation, capital investment, and planning and construction can be quickly resolved. The local government should play more roles in the school-enterprise cooperation, give full play to the supervision and guidance functions, build bridges for schools and enterprises, create a platform suitable for schoolenterprise cooperation, and actively promote the exchanges and cooperation between the two sides to cultivate local economic development. The high-quality talents have their own responsibility. They set up special organizations to regulate the cooperation, supervise the responsibilities and obligations between schools and enterprises, ensure the orderly cooperation and exchanges, and proceed smoothly.

\section{Conclusion}

Non-capital city colleges have unique social functions. In recent years, non-capital city colleges have focused on local economic development, adhered to the focus on personnel training, and raised the quality of education as the fundamental, continuously improved conditions for running schools, effectively increased reforms and innovations, and improved their ability to run schools and education. The talented, employable and skilled talents have provided important talent guarantees and intellectual support for local economic, social, and cultural construction, and have made important contributions to local economic development and social progress. However, non-capital city colleges still have such problems that cannot be avoided in their development. We should stick to the problem-oriented principle, take the analysis and resolution of issues as an opportunity, seize opportunities, deepen reforms, and make innovations in higher education in the new era. 


\section{References}

[1] Xie Yanlong. Current situation and strategy of China's Higher Education Management System [J]. Journal of Guangdong University of Technology, 2015, 5:5-8.

[2] Liu Ning. Research on the Training Strategy of Applied Talents in Local Colleges [J]. Journal of High Education, 2017, 3:150-151.

[3] Liu Yaohui, Yu Fang, Duan Sheng. Research on the "Double Teacher" Evaluation Standard for Information Specialty in Local Universities[J]. Computer Education, 2017, 7: 92-95.

[4] Cao Yumin. The Influence of Regional Culture on the Features of Running Local Colleges [J]. Jiangsu Higher Education, 2010, (6).

[5] Liu Liusheng, Wang Fei. Cultural Guidance: The Return and Approach of University Functions [J]. China Higher Education, 2010(5):28.

[6] Li Guoyi, Wang Weiyi. Thinking on the Optimization of Applied Talents Training Model in Local Universities [J]. Educational Exploration, 2015, 6: 67-71.

Project Information: Research Project on Major Problems in Higher Education Management in Shaanxi Province "Study on Supporting Non-Capital City Colleges Construction and Development Policy”, project number: 2016ZH15 